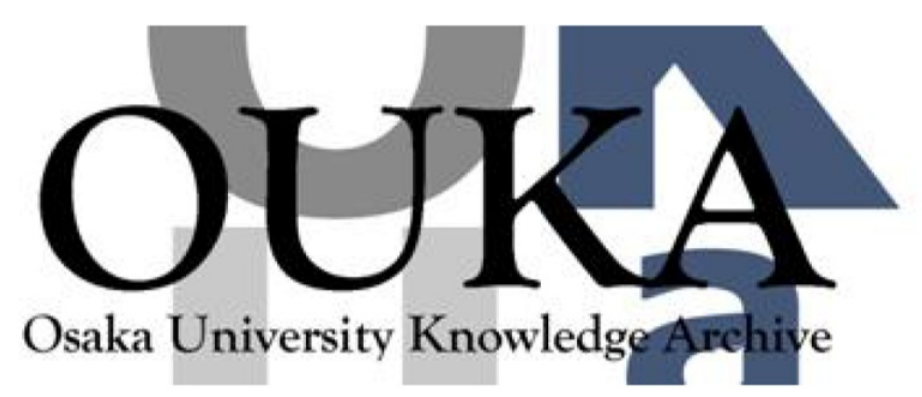

\begin{tabular}{|c|l|}
\hline Title & $\begin{array}{l}\text { Wavelet packet transform for Rms and power } \\
\text { measurements }\end{array}$ \\
\hline Author(s) & $\begin{array}{l}\text { Hamid, Effrina Yanti; Kawasaki, Zen-ichiro; } \\
\text { Mardiana, Redy }\end{array}$ \\
\hline Citation & $\begin{array}{l}2001 \text { Power Engineering Society Summer Meeting. } \\
\text { Conference Proceedings. } 3 \text { p. 1243-p. 1245 }\end{array}$ \\
\hline Issue Date & $2001-07$ \\
\hline oaire:version & VoR \\
\hline URL & $\begin{array}{l}\text { https://hdl. handle. net/11094/3237 } \\
\text { ब2001 IEEE. Personal use of this material is } \\
\text { permitted. However, permission to } \\
\text { reprint/republish this material for advertising } \\
\text { or promotional purposes or for creating new } \\
\text { collective works for resale or redistribution } \\
\text { to servers or lists, or to reuse any } \\
\text { copyrighted component of this work in other } \\
\text { works must be obtained from the IEEE. }\end{array}$ \\
\hline rights \\
\hline Note & \begin{tabular}{l} 
\\
\hline
\end{tabular} \\
\hline
\end{tabular}

Osaka University Knowledge Archive : OUKA

https://ir. Library. osaka-u. ac. jp/

Osaka University 


\title{
Wavelet Packet Transform for Rms and Power Measurements
}

\author{
Effrina Yanti Hamid, Zen-Ichiro Kawasaki, Member, IEEE, and Redy Mardiana, Member, IEEE
}

\begin{abstract}
This paper proposes an approach based on wavelet packet transform (WPT) for root mean square (rms) and power measurements. The algorithm can simultaneously measure the distribution of the rms and power with respect to individual frequency bands from the wavelet coefficients associated with each voltage current pair. The advantage of the WPT is that it can decompose a waveform into uniform frequency bands, which are important for identification of harmonic components and measurement of harmonic parameters. The algorithm is validated using simulated waveforms.
\end{abstract}

Index Terms-- Power, RMS, Harmonic, Wavelets, Wavelet Packet.

\section{INTRODUCTION}

$\mathrm{P}$ OWER quality is becoming an issue of increasing concern both to utilities and their customers. One of the major power system problems is steady-state waveform distortion due to harmonics. Harmonics are produced by variable speed drives, arc furnaces, personal computers, and other non-linear devices. Since harmonics can severely degrade the performance of power system equipment, it is necessary to always monitor their parameters such as voltage, current, and power [1], [2].

There has not been much work on applying wavelet transform for $\mathrm{rms}$ and power measurements. The discrete wavelet transform (DWT) algorithm for $\mathrm{rms}$ and power measurements has first been introduced in the literature [3]. The results show that the discrete wavelet-based algorithm could quantify rms and power of several harmonics within each frequency band. However, the waveform decomposition results using the DWT provide non-uniform frequency bands. For instance, at a higher level of decomposition, the frequency band becomes wider. As a result, frequency bands at the higher levels contain more harmonic components that those at lower levels. Therefore, the discrete wavelet-based algorithm can not measure the rms and power of individual harmonic components [3]. In practice, it is important to be able to identify the rms and power of individual harmonic components in order to know the sources and thus to eliminate their effects [1], [2].

To overcome the limitation, the DWT algorithm is

E.Y. Hamid, Z-I. Kawasaki, and R. Mardiana are with the Department of Electrıcal Engıneering, Osaka University, Suita, Osaka 565, Japan (e-mail: effrina@pels.pwr.wng.osaka-u.ac.jp). expanded to WPT algorithm in this study. Similarly to the DWT algorithm, in the WPT algorithm the input waveform is decomposed into wavelet coefficients, and frequency separation is achieved using a wavelet filter. The advantage of the WPT is that it can decompose a waveform into uniform frequency bands, so that this WPT algorithm has a capability to measure rms and power of individual harmonic components. In the wavelet transform, there are many types of wavelet filters. Here, we simply select the Vaidyanathan filter because of its good frequency selectivity [4].

\section{WAVELET PACKET TRANSFORM}

While detail mathematical background of WPT can be found in [4], a brief summary is given in this section. Wavelet packet transform is a direct expansion from the pyramid (or DWT) tree algorithm to a full binary tree. In the DWT algorithm the detail coefficients (or the output from high-pass filtering) are not used for further decomposition, only the approximation coefficients (or the output from low-pass filtering) at each level are treated to yield further approximation and detail coefficients. In the WPT algorithm, both the detail and approximation coefficients are decomposed into lower level to produce further coefficients (hereafter, both detail and approximation coefficients are called wavelet coefficients).

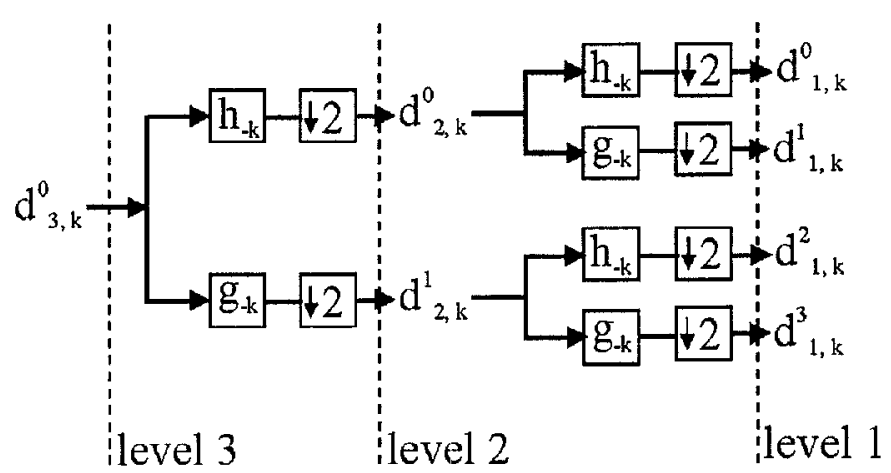

Fig. I. Wavelet packet decomposition with successive filterings and downsamplings.

Wavelet packet decomposition is depicted in Fig. 1. Let the original waveform has $2^{N}$ sampling points. The wavelet coefficient at the level $j$ th, $k$ th sampling point and node $i$ th is $d_{j, k}^{i}$, where $i=0,2, \ldots, 2^{N-j}-2$ (even) and $j=1,2, \ldots, N$. This 
coefficient is obtained by convolving the sequence $d^{i / 2}{ }_{j+1, k}$ with low-pass filter $h_{-k}$, and then downsampling by a factor of two. Similary, the wavelet coefficient at node $i=1,3, \ldots, 2^{N-j}$. 1 (odd) is obtained by convolving the sequence $d^{(i-1) / 2}{ }_{j+1, k}$ with high-pass filter $g_{-k}$ and downsampling by a factor of two. Number of nodes at level $j$ is $2^{N-j}$, and the node at level $N$ is the original waveform. The filters $h_{-k}$ and $g_{-k}$ are a pair of conjugate mirror filters (QMF), meaning both filters use the same set of coefficients, but with alternating signs and in reversed order. The Vaidyanathan filter is used in this study, and the filter coefficients can be found in [4].

The time resolution of $d_{j, k}^{i}$ is now half that of $d_{j+1, k}^{i}$ due to the downsampling. As a result, if $d_{j+1, k}^{i}$ has $2^{j+1}$ sampling points $\left(k=1,2, \ldots, 2^{j+1}\right)$ for the entire observation period, then $d_{j, k}^{i}$ will have $2^{j}$ sampling points $\left(k=1,2, \ldots, 2^{j}\right)$ for the same observation period. Each node at level $j$ has $2^{j}$ sampling points or wavelet coefficients.

\section{RMS AND POWER MEASUREMENTS}

The derivation of both the rms and power equations using discrete wavelet-based algorithm was proved in [3]. The following equations are extended forms from the DWT algorithm. The measurements consist of $I_{r m s}, V_{r m s}$, and power $(P)$. The definitions of these parameters are as follows (IEEE Std. 100-88):

$I_{r m s}=\sqrt{\frac{1}{T} \int_{0}^{T} i_{t}^{2} d t}, \quad V_{r m s}=\sqrt{\frac{1}{T} \int_{0}^{T} v_{t}^{2} d t}, \quad P=\frac{1}{T} \int_{0}^{T} i_{t} v_{t} d t$,

where $i_{t}$ and $v_{t}$ are respectively the analog current and voltage waveforms which are periodic during the observation period $T$. In practice, the analog waveforms are digitized. Here, $i_{n}$ and $v_{n}$ will be the digitized waveforms of $i_{t}$ and $v_{t}$, respectively, with $n=0,1, \ldots, 2^{N}-1$ ( $N$ is integer).

\section{A. Rms Calculations}

Rms of current or voltage in wavelet domain can be written as follows:

$$
\begin{aligned}
& I_{r m s}=\sqrt{\frac{1}{T} \int_{0}^{T} i_{t}^{2} d t} \cong \sqrt{\frac{1}{2^{N}} \sum_{n=0}^{2^{N}-1} i_{n}^{2}} \\
& =\sqrt{\frac{1}{2^{N}} \sum_{i=0}^{2^{N-j}} \sum_{k=0}^{2^{j}-1}\left(d_{j, k}^{i}\right)^{2}}=\sqrt{\sum_{i=0}^{2^{N-j}-1}\left(I_{j}^{i}\right)^{2}}, \\
& V_{r m s}=\sqrt{\frac{1}{T} \int_{0}^{T} v_{l}^{2} d t} \cong \sqrt{\frac{1}{2^{N}} \sum_{n=0}^{2^{N}-1} v_{n}^{2}} \\
& =\sqrt{\frac{1}{2^{N}} \sum_{i=0}^{2^{N-j}-1} \sum_{k=0}^{2^{j}-1}\left(d^{* i}{ }_{j, k}\right)^{2}}=\sqrt{\sum_{i=0}^{2^{N-j}-1}\left(V_{j}^{i}\right)^{2}},
\end{aligned}
$$

where $d_{j, k}^{i}$ and $d^{* i, k}$ are wavelet coefficients of $i_{n}$ and $v_{n}$, respectively. $I_{j}^{i}$ and $V_{j}^{i}$ are respectively the rms of current and voltage for the frequency band at node $i$ and level $j$. In the WPT algorithm, only the wavelet coefficients at a certain level $j$ are used for the rms and power calculations.

\section{B. Power Calculation}

The power calculation in wavelet domain is simply by multiplying the wavelet coefficients of current to those of voltage for every node at the same level, as follows:

$$
\begin{aligned}
& P=\frac{1}{T} \int_{0}^{T} i_{t} v_{t} d t \cong \frac{1}{2^{N}} \sum_{n=0}^{2^{N}-1} i_{n} v_{n} \\
& =\frac{1}{2^{N}} \sum_{i=0}^{2^{N-j}} \sum_{k=0}^{2^{j}-1} d_{j, k}^{i} d^{* i}{ }_{j, k}=\sum_{i=0}^{2^{N-j}-1} P_{j}^{i},
\end{aligned}
$$

where $P_{j}{ }_{j}$ is the power of frequency band at node $i$ and level $j$.
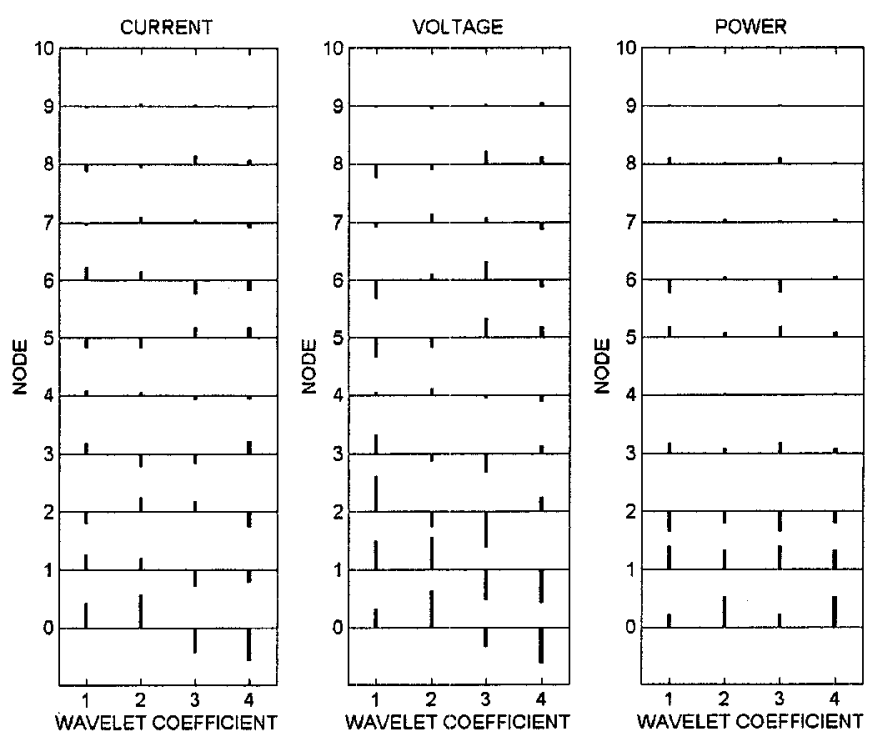

Fig. 2 Wavelet coefficients of the current, voltage, and power at level 2 for the first eleven nodes. The rest of nodes have very small or zero coefficients. (The ratios of node 0 and the other nodes are respectively 1:5, 1:5, and 1:50 for current, voltage, and power.)

\section{Evaluation}

To test the performance of power measurements using the WPT algorithm, simulated current and voltage waveforms will be analyzed. Each waveform has $128(N=7)$ sampling points per $60-\mathrm{Hz}$ fundamental cycle and contains first, third, fifth, seventh, eleventh, thirteenth, and seventeenth harmonics (odd integer harmonics), as follows:

$$
\begin{aligned}
& i(t)=1.0 \times \sqrt{2} \sin \left(2 \pi 60 t+10^{\circ}\right)+0.1 \times \sqrt{2} \sin \left(2 \pi 180 t+20^{\circ}\right) \\
& +0.08 \times \sqrt{2} \sin (2 \pi 300 t)+0.08 \times \sqrt{2} \sin \left(2 \pi 420 t+30^{\circ}\right) \\
& +0.07 \times \sqrt{2} \sin \left(2 \pi 660 t+45^{\circ}\right)+0.08 \times \sqrt{2} \sin \left(2 \pi 780 t+120^{\circ}\right) \\
& +0.05 \times \sqrt{2} \sin \left(2 \pi 1020 t+45^{\circ}\right) \\
& \text { and } \\
& v(t)=1.0 \times \sqrt{2} \sin (2 \pi 60 t)+0.2 \times \sqrt{2} \sin \left(2 \pi 180 t+30^{\circ}\right) \\
& +0.2 \times \sqrt{2} \sin \left(2 \pi 300 t+150^{\circ}\right)+0.1 \times \sqrt{2} \sin \left(2 \pi 420 t+60^{\circ}\right) \\
& +0.1 \times \sqrt{2} \sin \left(2 \pi 660 t+20^{\circ}\right)+0.1 \times \sqrt{2} \sin (2 \pi 780 t) \\
& +0.08 \times \sqrt{2} \sin \left(2 \pi 1020 t+45^{\circ}\right) .
\end{aligned}
$$


TABLE I

COMPARISON RESULTS BETWEEN CALCULATED AND TRUE VALUES OF RMS AND POWER MEASUREMENTS. (THE VALUES OF RMS AND POWER FOR THE REST OF NODES ARE VERY SMALL OR ZERO.)

\begin{tabular}{|c|c|c|c|c|c|c|c|c|}
\hline Node & $\begin{array}{c}\text { Frequency } \\
\text { Band (Hz) }\end{array}$ & $\begin{array}{c}\text { Harmonic } \\
\text { Component }\end{array}$ & $\begin{array}{c}\text { True } \\
\text { Irms }\end{array}$ & $\begin{array}{c}\text { True } \\
\text { Vrms }\end{array}$ & $\begin{array}{c}\text { True } \\
\text { Power }\end{array}$ & $\begin{array}{c}\text { Calculated } \\
\text { Irms }\end{array}$ & $\begin{array}{c}\text { Calculated } \\
\text { Vrms }\end{array}$ & $\begin{array}{c}\text { Calculated } \\
\text { Power }\end{array}$ \\
\hline 0 & DC-120 & $1^{\text {st }}$ & 1.0000 & 1.0000 & 0.9848 & 1.0000 & 1.0000 & 0.9848 \\
\hline 1 & $120-240$ & $3^{\text {rd }}$ & 0.1000 & 0.2000 & 0.0197 & 0.0941 & 0.2130 & 0.0197 \\
\hline 2 & $240-360$ & $5^{\text {th }}$ & 0.0800 & 0.2000 & -0.0139 & 0.0870 & 0.1858 & -0.0139 \\
\hline 3 & $360-480$ & $7^{\text {th }}$ & 0.0800 & 0.1000 & 0.0069 & 0.0756 & 0.0945 & 0.0062 \\
\hline 4 & $480-600$ & $9^{\text {th }}$ & 0 & 0 & 0 & 0.0263 & 0.0329 & 0.0008 \\
\hline 5 & $600-720$ & $11^{\text {th }}$ & 0.0700 & 0.1000 & -0.0063 & 0.0691 & 0.1050 & 0.0069 \\
\hline 6 & $720-840$ & $13^{\text {th }}$ & 0.0800 & 0.1000 & 0.0040 & 0.0795 & 0.0935 & -0.0044 \\
\hline 7 & $840-960$ & $15^{\text {th }}$ & 0 & 0 & 0 & 0.0260 & 0.0417 & 0.0011 \\
\hline 8 & $960-1080$ & $17^{\text {th }}$ & 0.0500 & 0.0800 & 0 & 0.0427 & 0.0683 & 0.0029 \\
\hline 9 & $1080-1200$ & $19^{\text {th }}$ & 0 & 0 & 0 & 0.0138 & 0.0173 & -0.0001 \\
\hline 10 & $1200-1320$ & $21^{\text {st }}$ & 0 & 0 & 0 & 0.0019 & 0.0013 & 0 \\
\hline 11 & $1320-1440$ & $23^{\text {rd }}$ & 0 & 0 & 0 & 0 & 0 & 0 \\
\hline 12 & $1440-1560$ & $25^{\text {th }}$ & 0 & 0 & 0 & 0 & 0 & 0 \\
\hline
\end{tabular}

First, both current and voltage waveforms are decomposed via the WPT algorithm as described in section II. All calculations use Matlab and WaveLab v.802 software package [5]. Next, only the wavelet coefficients at level 2 are used to calculate rms and power because each frequency band (or each node) at level 2 completely covers a respective harmonic component. Figure 2 shows the wavelet coefficients of current, voltage, and power for selected nodes. Level 2 has thirty-two nodes and each node has four coefficients. The wavelet coefficients at all nodes are then fed into (1) and (2) to compute the rms and power, respectively.

Table I shows the calculation results of using the WPT algorithm along with the true rms and power values. The true value is derived from analytical calculation. The table shows that the WPT algorithm can effectively compute the rms and power of each harmonic component. The total results of rms and power are the same in all cases. This proves that power measurements using WPT algorithm are valid. However, a small amount of rms and power leakage occurs at some nodes due to the roll-off characteristics of the low-pass and highpass filter pair.

\section{V.CONCLUSION}

A WPT-based approach has been proposed to improve the DWT-based approach for the rms and power measurements. The algorithm can separate harmonic components of power system waveforms and measure the rms and power of each harmonic component. The test using a simulated current voltage pair shows that the total results of $\mathrm{rms}$ and power are the same as those derived from analytical calculations. However, there are small errors to the rms and power at some nodes. These errors are due to the roll-off characteristics of the low-pass and high-pass filter pair. Hence, further study will be intended to find the suitable filter, which is able to minimize the error of rms and power measurement for all nodes.

\section{REFERENCES}

[1] S. Santoso,, W. M. Grady, E. J. Powers, J. Lamoree, and S. C. Bhatt " Characterization of Distribution Power Quality Events with Fourier and Wavelet Transforms ", IEEE Trans. Power Delivery, vol. 15, pp. 247 $254,2000$.

[2] V. L. Pham and K. P. Wong, "Wavelet transform-based algorithm for harmonic analysis of power system waveforms", IEE Proc. Gener. Transm. Distrib., vol. 146, pp. 249-255, 1999.

[3] W. K. Yoon and M. J. Devaney, "Power Measurement Using the Wavelet Transform", IEEE Trans. Instrum. Meas., vol. 47, pp. 1205-1209, 1998.

[4] M.V. Wickerhauser, "Adapted wavelet analysis from theory to software", IEEE Press, New York, USA, 1994, pp. 237-272 and pp. 443-462.

[5] D. L. Donoho, Software Package "WaveLab version .802" of Matlab Program, Aug. 1999.

\section{BIOGRAPHIES}

Effrina Yanti Hamid was born in Indonesia. She received the bachelor and master degrees in telecommuication engmeering from Bandung Institute of Technology, Indonesia in 1995 and 1998, respectively. Now, she is a Ph.D. student in the Department of Electrical Engineerıng, Osaka University. Her main interest is in signal processing and its application to power system.

Zen-Ichiro Kawasaki (M'72) was born in Japan. He received the B.S., M.S. and Dr. Eng. Degrees in communication engineering from Osaka University, Japan in 1973, 1975 and 1978, respectively. In 1989, he joined the Department of Electrical Engineering, Osaka University where he is currently a Professor. His current research interests are in signal processing, diagnosis techniques of power apparatus, and the electromagnetic of lightning discharges. Dr. Zen-Ichiro Kawasaki is a member of The Institute of Electrical and Electronics Engineers (IEEE), The Institute of Electrical Engineers of Japan (IEEJ), American Geophysical Union (AGU), and The Society of Atmospheric Electricity of Japan (SAEJ).

Redy Mardiana (M'99) was born in Indonesia. He received the bachelor and master degrees in electrical engineering from Bandung Institute of Technology, Indonesia in 1992 and 1997, respectively. From 1993 to 1994, he got a training fellowship in the Forschungzentrum $\Omega^{\prime \prime}\{u\}$ lich, $\Omega^{\prime \prime}\{u\}$ lich, Germany. Now, he is pursuing a Ph.D. degree in the Department of Electrical Engineering, Osaka University. His main interest is in lightning detection and its application to power system. He is a student member of The IEEE. 